\title{
Anti-IL-5 immunomodulates the effect of Staphylococcus aureus enterotoxin on T cell response in nasal polyps
}

\author{
S. Langier ${ }^{1}$, R. Landsberg ${ }^{2,3}$, K. Sade ${ }^{1}$, S. Kivity ${ }^{1}$ \\ 1 Allergy and Clinical Immunology Unit, Tel Aviv University, Tel Aviv, Israel \\ 2 The Ear Nose and Throat Department, Tel Aviv Medical Center, Sackler School of Medicine, Tel Aviv Uni- \\ versity, Tel Aviv, Israel \\ 3 Assuta Medical Center, Tel Aviv, Israel
}

SUMMARY

\begin{abstract}
Background: Chronic rhinosinusitis with nasal polyps (CRSwNP) is a Thelper-2 (Th2) inflammatory disease with considerable impact on the quality of life. Staphylococcus aureus enterotoxins (SAES) can induce andlor amplify inflammation. In this study, we investigated the immunomodulatory effect of SAEs on cytokine production by $T$ cell obtained from nasal polyps (NPS) and examined whether neutralizing interleukin 5 (IL-5) can reverse the immunological effect mediated by those toxins.
\end{abstract}

Methods: NP tissues were obtained from all patients who underwent endoscopic sinus surgery for CRSwNP. NP cells were isolated and stimulated in vitro with SAEs in the presence or absence of anti-IL-5. Flow cytometry (FACS) analyses were performed to measure specific T lymphocyte cytokine production.

Results: Seventeen patients (mean age 48 years) were enrolled. SAEs significantly increased the IL-4, IL-5 (Th2) and interferon (INF)- $\gamma$ (Th1) cytokines released from $T$ lymphocytes of NPS. The addition of anti-IL-5 suppressed IL-4 and INF- $\gamma$ release, which was most evident on NP tissue with high basal levels of IL-5.

Conclusions: Neutralizing IL-5 is a potential therapeutic modality in patients with NPs, the effect of which is dependent on IL-5 levels.

Key words: Interleukin-5, nasal polyps, Staphylococcus aureus enterotoxins, T cells, cytokines

\section{INTRODUCTION}

Chronic rhinosinusitis with nasal polyps (CRSwNP) is characterized by edematous masses in nasal and paranasal cavities, leading to nasal obstruction, rhinorrhea, loss of smell, headache and reduced overall well-being. NPs are believed to be a multifactorial disease frequently associated with asthma and occasionally associated with aspirin sensitivity ${ }^{(1)}$. The trigger and mechanisms underlying the development of NPs remain unknown. Although allergy has long been presumed as being the cause of NPs ${ }^{(2)}$, supported by the high eosinophil infiltrate, high levels of IgE and IL-5 in NP tissues ${ }^{(3)}$, this association was challenged in the 1970s when a retrospective study demonstrated that more NPs were found in non-atopic individuals than in atopic ones ${ }^{(4)}$. It was also demonstrated that the diagnosis of atopy based on positive skin-prick tests to inhalant allergens was not related to total and specific immu- noglobulin E (IgE) antibodies in NPs ${ }^{(2)}$. In addition, there was no relationship between atopy and the levels of interleukin (IL) 5, IL-4, eotaxins, leukotrienes or eosinophil infiltrate in tissue from NPs. Staphylococcus aureus (SA) enterotoxins (SAEs) were recently proposed as a cause of the amplification of the inflammation responsible for NP pathology. SA and polyclonal IgE specific for SAEs were found in about one-half of the specimens of NPs ${ }^{(5-7)}$. Additional data supporting this theory came from a report that an analysis of supernatants of cultures of NP tissue stimulated with SAE demonstrated an increase in pro-inflammatory Th1 and Th2 cytokines ${ }^{\left({ }^{8}\right)}$.

Given the important $\mathrm{T}$ helper 2 (Th2) inflammation in NP tissues, with high levels of local IL-5, IL-4, IL-13, eosinophil infiltrate and total and specific immunoglobulin E (IgE), (3), together with the evidence showing that treatment of the eosi- 
nophil-infiltrated tissue with neutralizing anti-IL-5 monoclonal antibodies induced eosinophil apoptosis and decreased tissue eosinophilia ${ }^{(9)}$, it seems logical that blocking Th2 inflammation can be a promising therapeutic option in NP management. Gevaert et al., ${ }^{(10)}$ used anti-IL-5 to treat patients with NPs and showed significant clinical improvement in one-half of the patients as well as a reduction in the number and function of eosinophils. The levels of nasal IL-5 predicted the response to the treatment: the higher the level, the better the response. However, that study did not explain the mechanisms behind the response.

The aim of this study was to investigate the influence of SAEs on $\mathrm{T}$ cell cytokine production in NPs and to determine whether neutralizing IL-5 can reverse the immunological effect mediated by those toxins.

\section{MATERIALS AND METHODS \\ Patients}

An ear nose and throat specialist carried out the initial evaluations of all the patients. They also underwent computerized tomographic (CT) scans and rhinoscopy, which demonstrated sinus disease in addition to NPs. All endoscopic sinus surgeries to remove NPs were carried out under general anesthesia. Tissue samples were obtained from patients visiting the Tel Aviv Medical Center and the Assuta Medical Center between February and December of 2010, and immediately submerged in PBS and processed. The samples were also examined by a pathologist, who confirmed the diagnosis of NP.

All the patients signed an informed consent form following the approval of the ethics committees of both medical centers.

\section{Obtaining NP dispersed cells (NPDCs)}

NPDCs were isolated from NPs by means of enzymatic digestion. The tissue was minced and incubated in the presence of $1.5 \mathrm{mg} / \mathrm{mL}$ collagenase, $0.75 \mathrm{mg} / \mathrm{mL}$ hyaluronidase and $0.05 \mathrm{mg} / \mathrm{mL}$ DNAse (all obtained from Sigma, USA) diluted in RPMI 1640 (Biological Industries, Beit Haemek, Israel) medium supplemented with $2 \mathrm{mM}$ glutamine, $100 \mathrm{U} / \mathrm{ml}$ penicillin, and $100 \mathrm{mg} / \mathrm{ml}$ streptomycin (complete RPMI) (all from Gibco, USA) and 2\% fetal calf serum (FCS, Biological Industries Beit Haemek, Israel) for one hour at $37^{\circ} \mathrm{C}$ in a shaking vortex mixer (Labnet, USA). The cell suspension was then filtered through a $70 \mu \mathrm{m}$ cell strainer (BD-Falcon, USA) to remove any undigested tissue, after which it was washed 3 times with RPMI. The pellet was suspended in culture medium (complete RPMI supplemented with 10\% FCS).

\section{Cell culture}

In flat-bottomed, 24-well culture plates (Corning, USA), $1 \mathrm{x}$ $10^{6} / \mathrm{ml}$ NPDCs were or were not stimulated with $100 \mathrm{ng} / \mathrm{mL}$ SA superantigens type B (SAB) ${ }^{(11)}$ (Sigma, USA) in the presence or absence of $1.5 \mu \mathrm{g} / \mathrm{mL}$ of anti-IL-5 (eBioscience, USA) or $1.5 \mu \mathrm{g} / \mathrm{mL}$ of the isotype control (rat anti-human IgG2a)
(eBioscience, USA) and incubated for $72 \mathrm{~h}$ at $37^{\circ} \mathrm{C}$ in the presence of $5 \% \mathrm{CO}_{2}$.

\section{Antibodies}

The antibodies used were: anti-human CD19 PE, anti-human CD3 FITC, anti-human CD3 PERCP Cy5.5, anti-human CD3 FITC, anti-human CD4 FITC, anti-human CD8 FITC, antihuman IL-4 PE, anti-human IL-5 PE, and anti-human INF- $\gamma$ PE (all from eBioscience, USA).

\section{Immunostaining}

For superficial (surface) staining, suspended cells $\left(10^{6}\right.$ cells $)$ were first incubated for $15 \mathrm{~min}$ with the appropriate antibodies diluted in $2 \%$ normal-rat-serum in phosphate-buffered solution (block solution, used to block Fc $\gamma$ II/III receptors). Then, this suspension was washed twice by two centrifugations (5 min, $450 \mathrm{~g}$ ) with block solution. An Ebioscience Intracellular Staining Kit (Ebioscience, USA) was used for intracellular staining, followed by incubation with the appropriate antibodies. The acquisition of the cells was done with FACSCalibur flow cytometry (FACS) equipment, and the analysis was performed on Cell Quest Pro software for Macintosh computers.

\section{Differential counts}

NPDCs were suspended in RPMI supplemented with 10\% FCS and placed in a cytocentrifuge cup already in place in a Shandon III cytocentrifuge (Shandon Southern Instruments, USA) for cytospin slides preparation. These slides were stained by Giemsa stain. Three-hundred cells were counted, and the results were expressed as a percentage of the total cell count.

\section{Plasma IL-5 detection}

IL-5 was measured in the plasma using a commercially available ELISA kit from R\&D Systems (USA). The sensitivity was $0.29 \mathrm{pg} / \mathrm{ml}$.

\section{Statistical analysis}

We performed a Wilcoxon non-parametric test to examine the influence of SAEs on IL-4, IL-5 and INF- $\gamma$ cytokines released from $\mathrm{T}$ lymphocytes of NPs. This test compares the levels of cytokines released before and after SAE stimulation. To test the effect of anti-IL-5 on cytokine release (IL-4 and INF- $\gamma$ ) after activation with SAEs and to determine whether this reaction is dependent upon the NP basal IL-5 levels of the tissue, we employed a mixed-model repeated-measures analysis of variance (ANOVA) with 1 between-subject variable (addition of anti-IL-5, i.e., SA vs. SA-anti IL-5), and 1 withinsubject variable (tissue basal level of IL-5, i.e., $>4 \%$ vs. $<4 \%$ ). Basal level effect slices was performed in each group of IL-5 for examining the difference in cytokine release prior to and following the addition of anti-IL-5. Significance was set at $\mathrm{p}<0.05$. 
A-

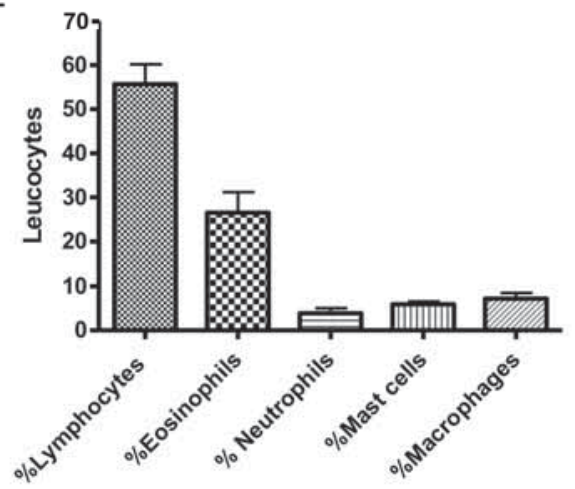

B-

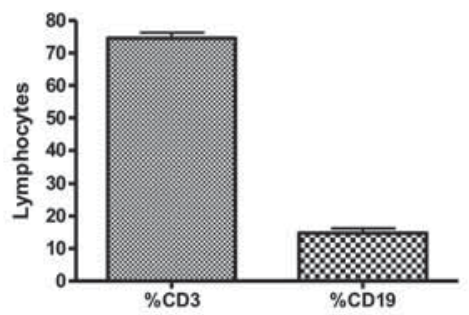

C-

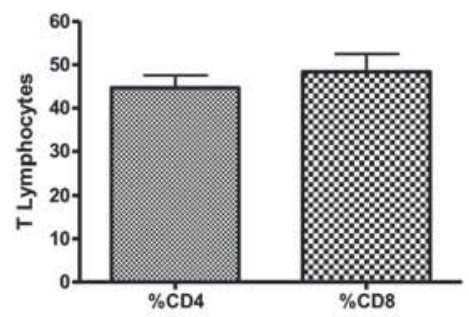

Figure 1. Percentage of leukocytes in nasal polyp (NP) tissue. (A) Differential count of leukocytes of dispersed cells in NPs. (B) FACS analysis of T (CD3+) and B (CD19) lymphocytes. (C) FACS analysis of CD4 and CD8 T lymphocytes subtypes.

A-

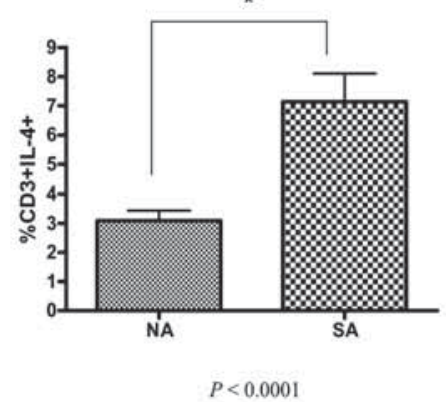

B-

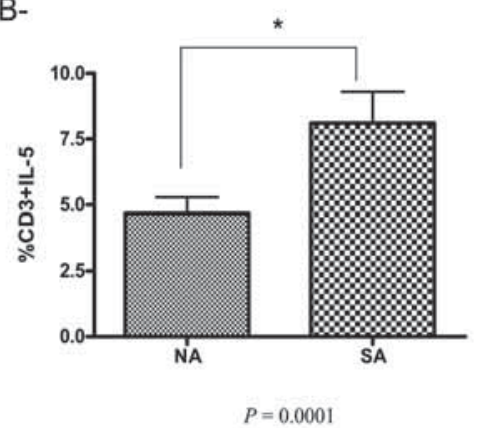

$P<0.0001$

\section{C-}

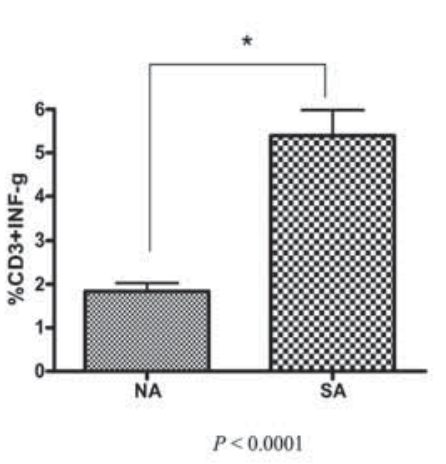

$P=0.000$

Figure 2. Increase in cytokine release in nasal polyp (NP) lymphocytes by Staphylococcus aureus enterotoxins (SAEs). NP tissues were collected directly after surgery. NP cells were dispersed by enzymatic digestion and cultured for 3 days in the presence or absence of $\mathrm{S}$. aureus superantigens type B (SABs). The cells were then collected, and the T lymphocyte cytokine release was measured by FACS. (A) INF- $\gamma$, (B) IL-4, (C) IL-5 T cell release. Non-activated, NP dispersed cells (NPDCs) culture in the presence of medium; SAB, NPDCs culture in the presence of medium and SAB.

A-

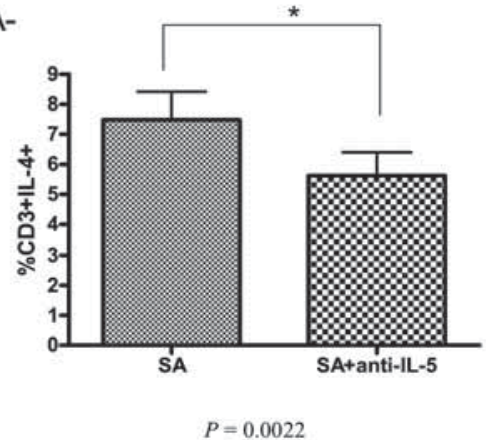

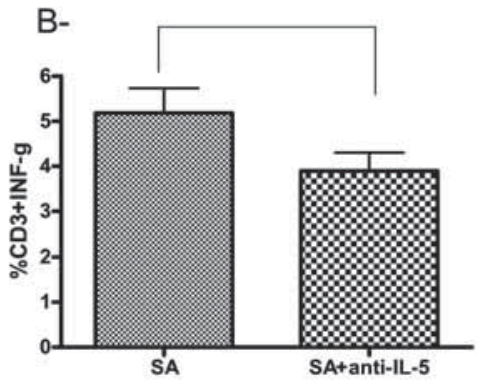

$P=0.148$

Figure 3. The effect of anti-IL-5 on NP T cells cytokine release. NP cells were dispersed by enzymatic digestion and activated with S. aureus superantigens type B (SABs) for 3 days in the presence or absence of anti-human IL-5. The cells were then collected, and the Tlymphocyte cytokine release was measured by FACS. (A) IL-4; (B) INF- $\gamma$. 
NPDs with CD3+IL-5+ >4\%

A-

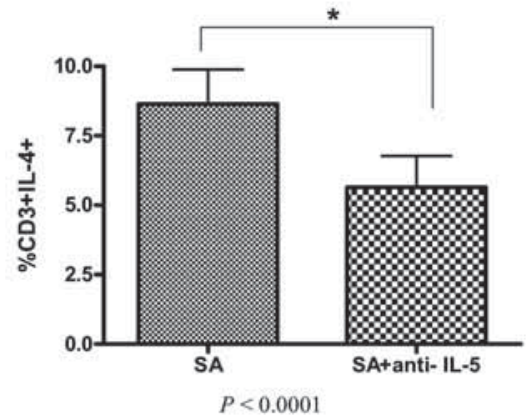

C-

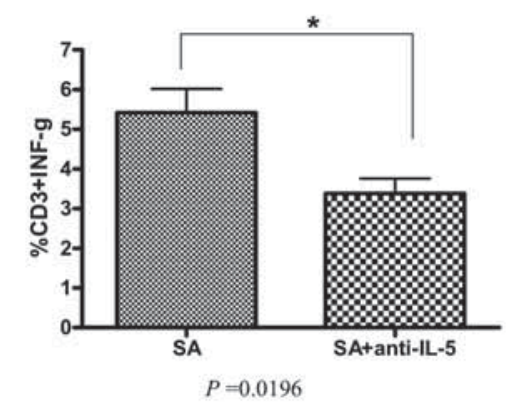

NPDs with CD3+IL-5+ $<4 \%$

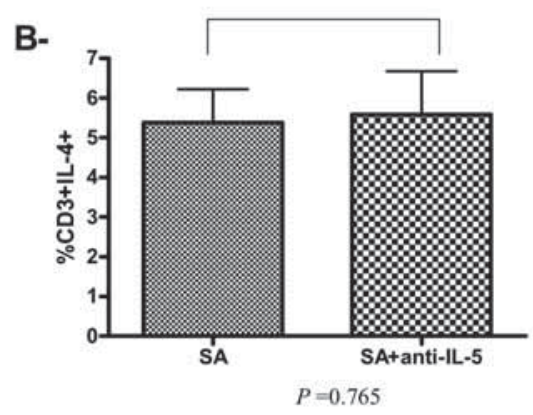

D-

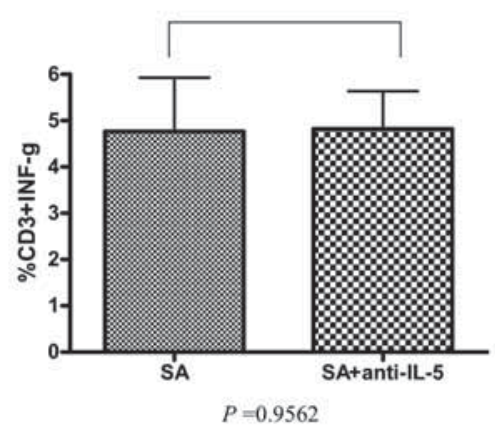

Figure 4. The need for high levels of basal IL-5 for the suppressive effect of anti-IL-5 on cytokine release. Nasal polyp (NP) tissues were collected directly after surgery. NP cells were dispersed by enzymatic digestion and activated with S. aureus superantigens type B (SABs) for 3 days in the presence or absence of anti-human IL-5. The cells were then collected, and the T lymphocyte cytokine release was measured by FACS. (A) IL-4 release in patients with basal IL-5 $>4 \%$, and (B) IL-4 release in patients with basal IL-5 $<4 \%$. (C) INF- $\gamma$ release in patients with basal IL-5 $>4 \%$. (D) INF- $\gamma$ release in patients with basal IL- $5<4 \%$. SAB, NP dispersed cells (NPDCs) in the presence of medium and SAB; SAB+anti-IL-5, NPDCs in the presence of medium and SAB and anti-IL-5.

\section{RESULTS}

\section{Patients}

Seventeen patients (12 males, 5 females) whose mean age was 48 years (age range 31 - 71 years) were enrolled. One patient had aspirin sensitivity.

\section{NP infiltrate}

The differential analysis of the NPDCs revealed considerable numbers of lymphocyte (mean 58.4\%) and eosinophil (mean $23.6 \%$ ) infiltrates (Figure 1A). Analysis of the lymphocytes by FACS revealed that the main subpopulation consisted of $\mathrm{T}$ cells (mean $74.6 \%$, of which there were $44.6 \%$ CD4 and $48.3 \%$ CD8; Figure 1C) compared with 14.8\% B cells (Figure 1B).

Increased T cell cytokine release in NP lymphocytes by SAEs NP tissues were collected from all 17 patients and NPDCs were cultured in vitro in the presence or absence of SAB. After three days of culture, the cells were collected and the cytokine release was analyzed by FACS. As shown in Figure 2 , the Wilcoxon sign rank test revealed a significant increase in IL-4, IL-5 and interferon INF- $\gamma$ release by NP T lymphocytes after SAE stimulation (Wilcoxon's statistic $=76.5 ; \mathrm{p}<0.0001$, Wilcoxon's statistic $=52.5 ; \mathrm{p}=0.0001$, Wilcoxon's statistic $=$ 75.5; $\mathrm{p}<0.0001$, respectively, Figure 2A, 2B and 2C).
The need for high levels of NP basal IL-5 for the suppressive effect of anti-IL-5 on cytokine release

To test the effect of anti-IL-5 on cytokine release after activation with SAEs, we added it to the culture and analyzed T cells IL-4 and INF- $\gamma$ cytokine release three days later. A mixed model repeated measures analysis of 14 of the 17 NP patients was used to examine the effect of anti-IL-5 on IL-4 T cell release. There was significant suppression in IL-4 release after the addition of anti-IL-5 ( $F=14.50 ; p=0.0022$; Figure 3A). Testing of effect slices indicated a significant difference in IL-4 means before compared to after anti-IL5 was added when the basal IL-5 level was $>4 \%(F=42.47$; $<<0.0001$; Figure 4A), but no such change was detected when the basal level was < $4 \%(F=0.09 ; p=0.7651 ;$ Figure $4 B)$.

Using the same mixed model repeated measures to analyze the effect of anti-IL-5 on INF- $\gamma$ T cell release revealed no significant changes after the addition of anti-IL-5 ( $F=2.34 ; p=0.1483$; Figure 3B). However, testing of effect slices revealed a significant change in INF- $\gamma$ after the addition of anti-IL5 when basal IL5 tissue levels were $>4 \%(F=6.94 ; p=0.0196$; Figure 4C), but not when they were $<4 \%(F=0.00 . p=0.9562$; Figure 4D). 
$1-$

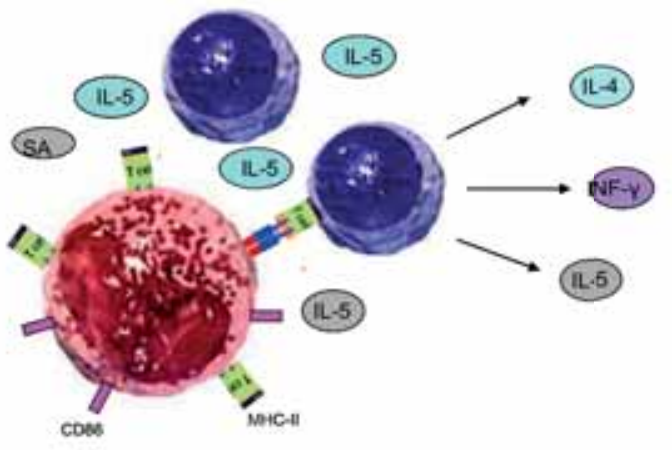

2-

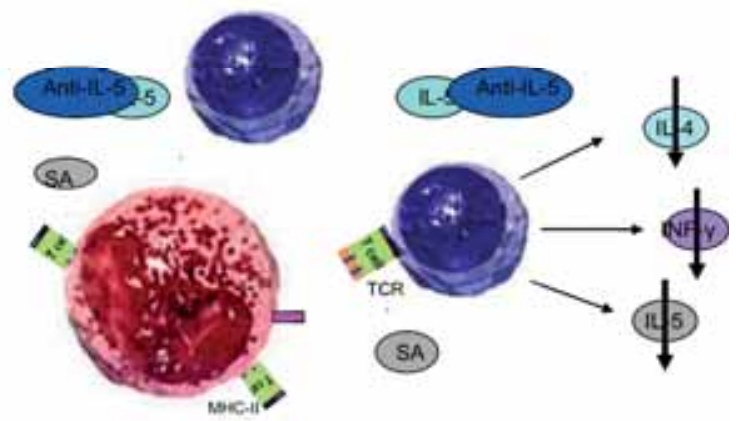

Figure 5. Schematic representation of the effect of eosinophils and IL-5 on antigen presentation and T cell activation. 1. The presence of IL-5 in culture, together with the antigen (SA), promotes activation of the eosinophils, which can now interact with $\mathrm{T}$ cells through MHCII and CD86 molecules, presenting antigens and activating those $\mathrm{T}$ cells to secrete cytokine (IL-4, IL-5 and INF- $\gamma$ ). 2. Blocking IL-5 inhibits this pathway, resulting in the decrease of IL- 4 and INF- $\gamma$ T cell secretion. SA, Staphylococcus Aureus enterotoxin; MHC-II, major histocompatibility complex II; TCR, T cell receptor; CD86, costimulatory molecule CD86.

The fact that there was a difference in INF- $\gamma$ release after the addition of anti-IL5 but the overall interaction effect was not significant may be explained by the small size of the groups in this study.

The addition of anti-IL-5 alone, i.e., without SAEs, in the culture produced cytokine release similar to the non-activated cells (data not shown). Moreover, the addition of isotype control of human anti-IL-5 antibodies together with the SAEs did not influence cytokine production by $\mathrm{T}$ lymphocytes (data not shown). Levels of IL-5 were not evaluated in this model because anti-IL-5 had been added into the culture. IL-5 was, however, tested in plasma, and there was no trace of it. NP IL-5 was not measured due to insufficient quantities of NP tissue.

\section{DISCUSSION}

The results of this study demonstrated that SABs promoted NP T cell activation by increasing the production of IL-4, IL-5 and INF- $\gamma$ cytokines (Figure 2). The addition of antiIL-5 suppressed the release of the analyzed cytokines (IL-4,
Figure $3 \mathrm{~A}$ and Figure $4 \mathrm{~A}$ and INF- $\gamma$, Figure 4C), and suppression was most evident when the level was $>4 \%$. In addition, plasma IL-5 was not detectable in those patients, confirming that the inflammation in this pathology was local and not systemic.

CRSwNP is a highly prevalent eosinophilic disease with a decisive impact on the health and quality of life of those who suffer from it ${ }^{(6,12)}$. The etiology and pathophysiology of CRSwNP remain poorly understood. It appears to be multifactorial disease. Th2 and Th1 cytokines (IL-4, IL-5, IL-13 and INF- $\gamma$ ) play a role in NP pathology (7). Most of the affected patients' NPs contain tissue eosinophilia, high levels of lymphocyte infiltrate with a predominance of $\mathrm{T}$ cells ${ }^{(13)}$, and high levels of tissue Th2 cytokines and IgE. Our analysis confirmed the predominance of a lymphocyte infiltrate with a prevalence of $\mathrm{T}$ cells (Figure 1A, B). We also analyzed the cytokine production specific to those cells before and after the desired stimulus. Eosinophilia (> 20\% eosinophil infiltrate) was present in 11 of our 17 study patients (Figure 1A).

The sinonasal cavities of NP patients were previously reported to be frequently colonized by SA ${ }^{(5)}$. Most SA bacteria can secrete protein enterotoxins that are able to activate $\mathrm{T}$ cells by promoting the crosslinking binding of the $\beta$-chain of $\mathrm{T}$ cell receptors to the major histocompatibility complex class II, independent of the antigen-specific groove. This can cause a sudden cytokine storm, which is the reason why those molecules are also called 'superantigens' ${ }^{(14)}$. Immunological responses against SAEs present in this tissue can induce and/ or amplify a pre-existing inflammation, including $\mathrm{T}$ cell activation and cytokine production, polyclonal activation of $\mathrm{B}$ cells, and eosinophil recruitment ${ }^{(15)}$, which can aggravate the clinical condition. Several groups support a role of SAEs in NPs. For example, SAEs have been directly detected in the nasal mucosa or mucus of $48 \%$ of patients with NPs, which is significantly more than the levels in patients with chronic rhinosinusitis without nasal polyps $(7 \%)$ and the lack of it in healthy individuals ${ }^{(5)}$. However, Heymans et al., screened 22 currently known enterotoxins genes and found no correlation with the presence of SAE and disease severity ${ }^{(16)}$. One possibility is that those NP patients respond in a different way to these antigens. In support of that reasoning, SA and polyclonal IgE specific for SAEs were found in about one-half of the NP specimens ${ }^{(5-7)}$. Gevaert et al., reported that the presence of IgE directed against SAEs was associated with higher rates of eosinophilic inflammation ${ }^{(17)}$. Others reported that the analysis of supernatants of cultures of NP tissue stimulated with SAB demonstrated an increase in pro-inflammatory, Th1 and Th2 cytokines ${ }^{(8)}$. In light of the potent effect of SAEs on NP inflammatory response and the large number of $\mathrm{T}$ cell infiltrates found in those tissues, we investigated the behavior of those lymphocytes when they were stimulated in vitro with SAEs. Our data demonstrated that the percentage of $\mathrm{T}$ cells that produce IL-4, IL-5 (Th2 cytokines) and INF- $\gamma$ 
(Th1 cytokine) increased significantly compared to the nonactivated cells following activation with SAEs. We concluded that SAEs non-specifically activate the T lymphocytes in NPs. Since NPs are known as being predominantly a Th2 inflammation, this stimulus probably amplified these responses in Th2-primed NPs. It remains unclear, however, what triggers the Th2 inflammation and the growth of NPs.

Although, there is no clear evidence of a direct effect of antiIL-5 on T cell cytokine production, given the essential role of $\mathrm{T}$ lymphocytes in immune responses and the significant $\mathrm{T}$ cell infiltrate observed in NP tissue (Figure 1), we assumed that it would be important to analyze the indirect effect of anti-IL-5 on T lymphocyte cytokine production.

Our results demonstrated that neutralization of IL-5 (the cytokine crucial for eosinophil maturation and attraction) resulted in suppression of T cell release of IL-4 and INF- $\gamma$ that had been induced by SAEs in NPDCs. The effect of antiIL-5 was more pronounced in NPs with high levels of IL-5. How can we explain those findings in light of the lack of a direct effect of IL-5 on T cell activation? Liu et al., reported that human airways and peripheral blood eosinophils stimulated in vitro with SAE enhanced Th1 and Th2 T cell cytokine production ${ }^{(18)}$. In addition, eosinophils were shown to be able to function as antigen-presenting cells (APCs) promoting Th2 ${ }^{(19)}$ and Th1 ${ }^{(20)} \mathrm{T}$ cell-mediated immune responses. Finally, in vitro stimulation of the eosinophils with antigen and IL-5 modulate the major histocompatibility complex II (MHC- II) and CD86 molecules ${ }^{(19,21,22)}$. These molecules interact with $\mathrm{T}$ cells, presenting antigens and activating them to secrete cytokine. These data indicate that anti IL-5 can indirectly modulate $\mathrm{T}$ cell function through eosinophils. We therefore suggest that the presence of IL-5 in culture, together with the antigen, promote activation of the eosinophils, which can now function as APC, activating T cells to secrete cytokine. Blocking IL-5 inhibits this pathway, resulting in the decrease of IL-4 and INF- $\gamma$ T cell secretion. This hypothesis is now presented schematically in Figure 5.

Clinically, our results are in agreement with those of Gevaert et al., who reported a good improvement in clinical score and decreased blood and nasal eosinophils in the individuals who presented with high basal IL-5 levels and were treated by systemic anti-IL-5 ${ }^{(10)}$. Furthermore, Simon et al., noted that treatment of the eosinophil-infiltrated tissue with neutralizing anti-IL-5 monoclonal antibodies induced eosinophil apoptosis and decreased tissue eosinophilia ${ }^{(9)}$. The data presented herein add to the understanding of the mechanism and condition in which anti-IL5 can modify and improve the inflammatory reaction responsible for the NP growth in those patients.

\section{CONCLUSION}

SAEs seem to contribute to the development and growth of NPs in patients with chronic rhinosinusitis. IL-5 is one of the cytokines involved in NP inflammation, and anti-IL-5 seems to be effective in suppressing the inflammation in patients who have high levels of IL-5. It is highly probable that other mechanisms are involved in this process, and further research for finding other modalities of treatment is warranted.

\section{ACKNOWLEDGMENT}

Esther Eshkol is thanked for editorial assistance.

\section{CONFLICT OF INTEREST}

None.

\section{REFERENCES}

1. Hamilos DL. Chronic rhinosinusitis patterns of illness. Clin Allergy Immunol. 2007; 20: 1-13.

2. Voegels RL, Santoro P, Butugan O, Formigoni LG. Nasal polyposis and allergy: is there a correlation? Am J Rhinol. 2001; 15: 9-14.

3. Bachert C, Gevaert P, Holtappels G, van Cauwenberge P. Mediators in nasal polyposis. Curr Allergy Asthma Rep. 2002; 2: 481-487.

4. Settipane GA, Chafee FH. Nasal polyps in asthma and rhinitis. A review of 6037 patients. J Allergy Clin Immunol. 1977; 59: 17-21.

5. Seiberling KA, Conley DB, Tripathi A, et al. Superantigens and chronic rhinosinusitis: detection of staphylococcal exotoxins in nasal polyps. Laryngoscope. 2005; 115: 1580-1585.

6. Bachert C, Gevaert P, Holtappels G, Johansson SG, van Cauwenberge $\mathrm{P}$. Total and specific IgE in nasal polyps is related to local eosinophilic inflammation. J Allergy Clin Immunol. 2001; 107: 607-614.

7. Bernstein JM, Ballow M, Schlievert PM, et al. A superantigen hypothesis for the pathogenesis of chronic hyperplastic sinusitis with massive nasal polyposis. Am J Rhinol. 2003; 17: 321-326.

8. Patou J, Gevaert P, Van Zele T, Holtappels G, van Cauwenberge P, Bachert C. Staphylococcus aureus enterotoxin B, protein A, and lipoteichoic acid stimulations in nasal polyps. J Allergy Clin Immunol. 2008; 121: 110-115.

9. Simon HU, Yousefi S, Schranz C, et al. Direct demonstration of delayed eosinophil apoptosis as a mechanism causing tissue eosinophilia. J Immunol. 1997; 158: 3902-3908.

10. Gevaert P, Lang-Loidolt D, Lackner A, et al. Nasal IL-5 levels determine the response to anti-IL-5 treatment in patients with nasal polyps. J Allergy Clin Immunol. 2006; 118: 1133-1141.

11. Okano M, Fujiwara T, Haruna T, et al. Prostaglandin E(2) suppresses staphylococcal enterotoxin-induced eosinophilia-associated cellular responses dominantly through an E-prostanoid 2-mediated pathway in nasal polyps. J Allergy Clin Immunol. 2009; 123: 868-874 e13.

12. Ling M, Hamilos DL. Are we closer to understanding the pathophysiology of chronic rhinosinusitis? Clin Exp Allergy. 2011; 41: 144-146.

13. Sanchez-Segura A, Brieva JA, Rodriguez C. T lymphocytes that infiltrate nasal polyps have a specialized phenotype and produce a mixed TH1/TH2 pattern of cytokines. J Allergy Clin Immunol. 1998; 102: 953-960.

14. Fraser JD, Proft T. The bacterial superantigen and superantigenlike proteins. Immunol Rev. 2008; 225: 226-243.

15. Bachert C, Zhang N, Patou J, van Zele T, Gevaert P. Role of staphylococcal superantigens in upper airway disease. Curr Opin Allergy Clin Immunol. 2008; 8: 34-38.

16. Heymans F, Fischer A, Stow NW, et al. Screening for staphylococcal superantigen genes shows no correlation with the presence or the severity of chronic rhinosinusitis and nasal polyposis. PLoS One 2010; 5: e9525.

17. Gevaert P, Holtappels G, Johansson SG, et al. Organization of secondary lymphoid tissue and local IgE formation to Staphylococcus aureus enterotoxins in nasal polyp tissue. Allergy. 2005; 60: 71-79. 
18. Liu LY, Mathur SK, Sedgwick JB, et al. Human airway and peripheral blood eosinophils enhance Th1 and Th2 cytokine secretion. Allergy. 2006; 61: 589-597.

19. Padigel UM, Lee JJ, Nolan TJ, Schad GA, Abraham D. Eosinophils can function as antigen-presenting cells to induce primary and secondary immune responses to Strongyloides stercoralis. Infect Immun. 2006; 74: 3232-3238.

20. Le Moine A, Surquin M, Demoor FX, et al. IL-5 mediates eosinophilic rejection of MHC class II-disparate skin allografts in mice. J Immunol. 1999; 163: 3778-3784.

21. Shi HZ. Eosinophils function as antigen-presenting cells. J Leukoc Biol. 2004; 76: 520-527.

22. Rizzo LV. Differential modulatory effect of IL-5 on MHC class II expression by macrophages and B cells. Braz J Med Biol Res. 1992; 25: 509-513.
Shmuel Kivity, MD

Allergy and Clinical Immunology Unit

Tel Aviv Medical Center

6 Weizman Street

Tel Aviv 64239

Israel

Tel: +972-3-6973734 (cell: 052-426-6899)

Fax: +972-3-6974601

E-mail: allergy@tasmc.health.gov.il

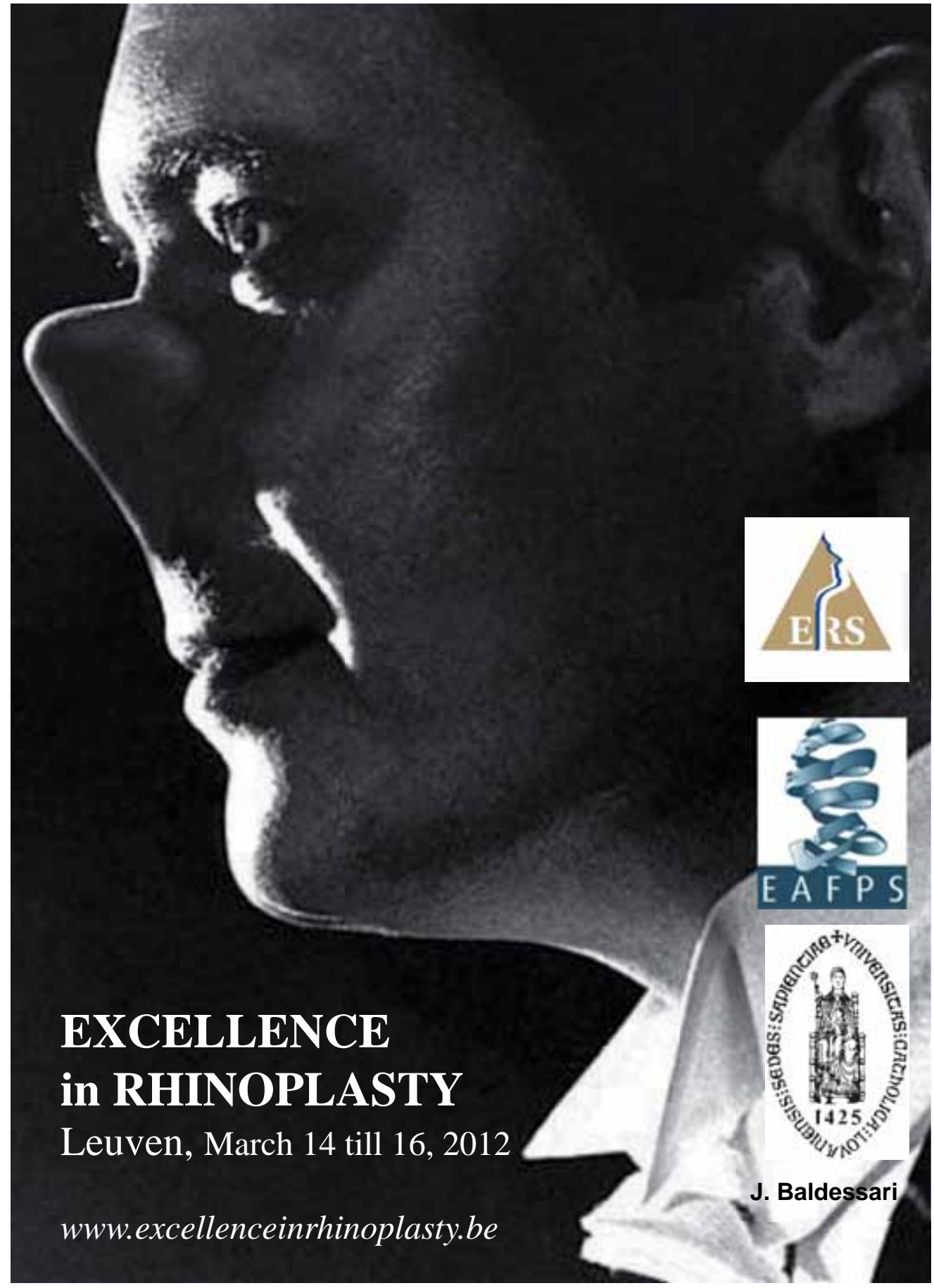

